\title{
Dynamic Modulation of Microglia/Macrophage Polarization by miR-124 after Focal Cerebral Ischemia
}

\author{
Somayyeh Hamzei Taj ${ }^{1}$ - Widuri Kho ${ }^{1}$ Markus Aswendt ${ }^{1}$ - Franziska M. Collmann ${ }^{1,2}$. \\ Claudia Green $^{1}$ - Joanna Adamczak ${ }^{1,3}$ - Annette Tennstaedt ${ }^{1}$ - Mathias Hoehn ${ }^{1,2,3}$
}

Received: 5 July 2016 / Accepted: 28 July 2016/Published online: 18 August 2016

(C) The Author(s) 2016. This article is published with open access at Springerlink.com

\begin{abstract}
Mononuclear phagocytes respond to ischemic stroke dynamically, undergoing an early anti-inflammatory and protective phenotype followed by the pro-inflammatory and detrimental type. These dual roles of microglia/macrophages suggest the need of subtle adjustment of their polarization state instead of broad suppression. The most abundant brainspecific miRNA, miR-124, promotes neuronal differentiation but can also modulate microglia activation and keeps them in a quiescent state. We addressed whether the intracerebral injection of miR-124 in a mouse model of ischemic stroke before or after the peak phase of the pro-inflammatory polarization modifies the pro-/anti- inflammatory balance. In the sub-acute phase, $48 \mathrm{~h}$ after stroke, liposomated miR-124 shifted the predominantly pro-inflammatory polarized microglia/macrophages toward the anti-inflammatory phenotype. The altered immune response improved neurological deficit at day 6 after stroke. When miR-124 was injected 10 days after stroke, the pro-/anti- inflammatory ratio was still significantly reduced although to a lower degree and had no effect on recovery at day 14 . This study indicates that miR-124 administration before the peak of the proinflammatory process of stroke is most effective in support
\end{abstract}

Electronic supplementary material The online version of this article (doi:10.1007/s11481-016-9700-y) contains supplementary material, which is available to authorized users.

Mathias Hoehn

mathias@sf.mpg.de

1 In-vivo-NMR Laboratory, Max Planck Institute for Metabolism Research, Gleuelerstrasse 50, D-50931 Köln, Germany

2 Department of Radiology, Leiden University Medical Center, Leiden, Netherlands

3 Percuros BV, Enschede, Netherlands of increasing the rehabilitation opportunity in the sub-acute phases of stroke. Our findings highlight the important role of immune cells after stroke and the therapeutic relevance of their polarization balance.

Keywords Stroke - Microglia/macrophage polarization . Pro-inflammatory and anti-inflammatory phenotypes . miRNA-124 $\cdot$ Neuroinflammation

\section{Introduction}

Ischemic stroke is a major cause of death and disability worldwide (Donnan et al. 2008), but presently available therapies only benefit a small fraction of all patients. In stroke, primary cell death is directly caused by ischemia, but is followed by secondary deterioration due to inflammatory response to the primary event (Dirnagl et al. 1999; Jin et al. 2010). Next to neutrophils, granulocytes and T-cells infiltrating at day 2-4 after stroke, the early recruitment of central nervous system (CNS) resident microglia and infiltrating macrophages at day 1-2 determines strongly the outcome of CNS repair (Hanisch and Kettenmann 2007). However, these cells have been shown to play a protective as well as a detrimental role. They promote CNS recovery by cleaning up cell debris and releasing a variety of trophic factors and cytokines that are important for neurogenesis, axonal regeneration, angiogenesis, and vascular repair (Thored et al. 2009; Kwon et al. 2013; Hu et al. 2015). On the other hand, microglia/macrophages can aggravate tissue damage and impair functional recovery (Ekdahl et al. 2003; Miron et al. 2013). This dual role has been described as a polarization between two phenotypes, i.e. the anti-inflammatory, protective state at early stages of ischemic stroke and the pro-inflammatory, detrimental state which dominates at later stages (Hu et al. 2012). The two 
activation phenotypes are mainly characterized by increased protein synthesis of anti-inflammatory mediators such as interleukin-10 (IL-10), IL-4, IL-13, and tumor growth factor $\beta$ (TGF- $\beta$ ), or pro-inflammatory mediators such as interferon $\gamma$ (IFN- $\gamma$ ), tumor necrosis factor $\alpha$ (TNF- $\alpha$ ), and IL-6 (Patel et al. 2013). In addition, pro-inflammatory factors, including iNOS, CD16, CD32, and CD86 are biomarkers of microglia/macrophages, which are regarded to be detrimental (Jia et al. 2016)(Hirai et al. 2013). In contrast, antiinflammatory factors Arg1, CD206, CCL22, Ym1/2 are the biomarkers of microglia/macrophages, which are considered to be beneficial (Hu et al. 2012) (Liu et al. 2013). Stroke induces a systemic immunodepression, which leads to infections such as pneumonia as a leading cause of death in patients after stroke. However, preventive antibiotic therapy and broad immune suppression failed to improve clinical outcome in stroke patients (Dirnagl et al. 2007; Hu et al. 2012). Therefore, we focused on a novel class of immune modulators with clinical potential to target specific immune cell phenotypes and modify their activation state.

A key regulator of microglia quiescence and modulator of macrophage activation is miR-124 (Ponomarev et al. 2011). Similar to small interfering ribonucleic acid (siRNA), microRNAs are small non-protein coding RNAs which bind to mRNAs and lead to their direct degradation and subsequent suppression of protein translation (Bartel 2009; Bi et al. 2009). The most abundant brain-specific microRNA, miR-124, has been shown to promote neuronal differentiation in neuronal progenitor cells (Makeyev et al. 2007; Visvanathan et al. 2007). miR-124 is expressed in microglia but not in peripheral monocytes and macrophages. It affects two main transcription factors CCAAT/enhancer binding protein alpha (C/EBP- $\alpha)$, and its downstream target PU.1, resulting in a switch from an activated state to a non-activated $\mathrm{CD} 45^{\text {low }}$ and major histocompatibility class (MHC) $\mathrm{II}^{\text {low }}$ state (Ponomarev et al. 2011). Peripheral administration of miR-124 in experimental autoimmune encephalomyelitis caused suppression of the disease by deactivation of macrophages and reduced activation of myelin-specific T-cells. Recently, we have been able to demonstrate that intracerebral administration of miR-124 after stroke has led to a clear shift of microglia/ macrophages into the anti-inflammatory phenotype, as recognized by the upregulation of the marker Arg-1. This treatment resulted in a significant correlation of Arg-1 upregulation with neuronal survival and with functional improvement (Hamzei Taj et al. 2016).

These earlier results motivated us to investigate in the present study how miR-124 affects the polarization ratio of proinflammatory (M1) and anti-inflammatory (M2) phenotypes in microglia/macrophages after stroke. Although we are aware of the description of the states with M1 and M2 being a simplified scheme, we decided to stick to this nomenclature as it best describes our intention of shifting the status from the pro- inflammatory, detrimental towards the anti-inflammatory, protective phase. For this purpose, we selected the widely used CD16/32 as a pro-inflammatory biomarker, which is implicated in cellular cytotoxicity (Bhatnagar et al. 2014), and the mannose receptor CD206 as an anti-inflammatory biomarker, which is involved in tissue recovery and function restoration (Perego et al. 2011). We show here for the first time that intracerebral miR-124 administration after stroke results in a significant increase of microglia/macrophages with M2 phenotype, paralleled by a decrease of the M1 phenotype. This substantial decrease of the M1:M2 ratio correlates strongly with functional outcome, thus indicating the therapeutic role for miR-124 in shifting the inflammatory cells into the antiinflammatory, protective M2 phenotype.

\section{Material and Methods}

\section{Experimental Groups}

The aim of this study was to investigate whether the intracerebral injection of miR-124 in a mouse model of ischemic stroke before or after the peak phase of M1 polarization modifies the M1/M2 balance. For this purpose, a total of 34 adult male C57BL6/N mice (11-12 weeks, 21-25 g; Janvier, Saint Berthevin Cedex, France) were randomly allocated to different experimental groups of miR-124 injection and survival times. Animals were housed under fixed circadian rhythm with ad libitum access to food and water. All surgical and scanning procedures were performed under Isoflurane anesthesia and core body temperature control. Stroke was induced in all mice via the middle cerebral artery occlusion (MCAO) model (cf. below). Successful ischemic stroke lesions were verified by magnetic resonance imaging (MRI) scans $48 \mathrm{~h}$ after MCAO, before intracranial miR-124 injection, and again before perfusion fixation at day 6 or day 14, respectively.

In Experiment 1, fifteen mice were subjected to MCAO and perfused 6 days later, divided into the following three groups: i) control mice exposed to stroke only $(n=5)$, ii) miR-treated mice which received an intracranial injection of liposomated miR-124 $48 \mathrm{~h}$ after MCAO $(n=5)$, and iii) mice receiving a liposomated random primer injection $48 \mathrm{~h}$ after MCAO ( $\mathrm{n}=5)$ as negative control.

In Experiment 2, fourteen mice were subjected to MCAO and perfused 14 days later, divided into the following three groups: i) control mice subjected to stroke only $(n=5)$, ii) miR-treated mice which received an intracranial injection of liposomated miR-124 $48 \mathrm{~h}$ after MCAO $(n=6)$, and iii) the negative control group that was injected with a liposomated random primer $48 \mathrm{~h}$ after $\mathrm{MCAO}(n=3)$.

In Experiment 3, stroke was induced in ten mice, and they were perfused 14 days later, divided into the following two groups: control mice subjected to stroke only $(n=5)$, and the 
miR-treated mice with liposomated miR-124 injection at day 10 post $\operatorname{MCAO}(\mathrm{n}=5)$.

\section{Middle Cerebral Artery Occlusion}

Transient occlusion of the right middle cerebral artery (MCAO) was induced with the intraluminal filament model, as described previously (Adamczak et al. 2014). Briefly, mice were initially anesthetized with $2 \%$ Isoflurane $\left(\mathrm{O}_{2}: \mathrm{N}_{2} \mathrm{O}\right.$, $30: 70 \%$ ) and were subcutaneously injected with $4 \mathrm{mg} / \mathrm{kg}$ Carprofen (Pfizer, Berlin, Germany). The body core temperature was controlled during surgery via a temperature-regulated heating pad (medres $\mathrm{GmbH}$, Cologne, Germany). The right common carotid artery (CCA), external carotid artery (ECA), and internal carotid artery (ICA) were presented. A silicon rubber-coated filament with the length of $20 \mathrm{~mm}$ and a tip diameter of $170 \mu \mathrm{m}$ (7017PK5Re, Doccol Corporation, Sharon, USA) was advanced into the ICA lumen until it blocked the origin of MCA. During 30 min occlusion time, mice were allowed to recover from anesthesia in a controlled heating box. Afterwards, the animals were re-anesthetized, the reperfusion was induced by filament withdrawal and the CCA was ligated. Following surgery, $\mathrm{NaCl}$ was subcutaneously injected in all animals twice daily until stabilization of body weight. Only the mice with cortico-striatal lesion, observed by MRI two days after MCAO, were selected for the experiments.

\section{Intracranial Injection of Liposomated miR-124}

T2-weighted MRI was used to determine the injection coordinates $48 \mathrm{~h}$ after MCAO. At the day of injection the miR-124 ( $2 \mu \mathrm{g}$ in $50 \mu \mathrm{l} \mathrm{PBS}$, PM10691 Applied Biosystems, Carlsbad, CA, USA) or the negative control of miR ( $2 \mu \mathrm{g}$ in $50 \mu \mathrm{l} \mathrm{PBS}$, AM17110; Applied Biosystems) was mixed with the transfection agent Lipofectamine 2000 (Invitrogen, Paisley, UK). Intracranial injection into the right striatum, ipsilateral to the lesion, was performed as described elsewhere (Aswendt et al. 2012). Briefly, mice were fixed in a stereotactic frame (Stoelting, Dublin, Ireland), and $2.0 \mu \mathrm{l}$ of the suspension containing $100 \mathrm{ng}$ of $\mathrm{miR}$ or random primer was injected with a Hamilton syringe (26G needle) at the following coordinates relative to bregma: $\mathrm{AP}+0.5$; $\mathrm{L}+1.4$; DV -2.4 with an infusion rate of $500 \mathrm{nl} / \mathrm{min}$. The needle was kept in place for another $5 \mathrm{~min}$ before removal.

\section{Functional Test}

To observe the different aspects of neurological functions, a set of behavioral tests was performed before and every 2 days after MCAO using the modified neurological deficit scores (mNDS), a modification of a previous report (Chen et al. 2001). The modified NDS (mNDS) has already been described recently (Hamzei Taj et al. 2016). In short, it consists of a set of motor tests (muscle status and abnormal movement), sensory tests (tactile, and proprioceptive), and reflex tests on a scale of $0-16$. One point was given for the failure of a performed test or for the loss of a tested reflex. Thus, higher scores indicate higher severity of ischemia.

\section{Magnetic Resonance Imaging}

MRI was performed using a 9.4 T Biospec animal MRI system with a $20 \mathrm{~cm}$ horizontal bore magnet (Bruker BioSpin, Ettlingen, Germany) equipped with actively shielded gradient coils (BGA9S, 750 mT/m, Bruker BioSpin), using ParaVision 5 software. Radio frequency (RF) transmission was achieved with a $112 / 72 \mathrm{~mm} \mathrm{od} /$ id mouse resonator (Bruker) while signal was received with a dedicated quadrature mouse head surface coil (Bruker BioSpin). Mice were anesthetized with $2 \%$ Isoflurane in a 30/70 oxygen/air mixture and placed in an animal holder (Bruker) using a tooth bar and ear bars for stable positioning. Respiration rate was monitored using a pressure sensitive pad placed underneath the mice. The physiological status of the animal was monitored with DASYlab software (National Instruments, Austin, TX, USA). The body core temperature was monitored with a rectal probe and was kept constant at $37 \pm 1.0{ }^{\circ} \mathrm{C}$ using a water blanket connected to a feedback controlled automated temperature control unit (medres, Cologne).

Tripilot gradient-echo scans were used for definite positioning of the mouse head in the magnet. For lesion visualization, T2-weighted images were acquired with a multi-slice multi-echo (MSME) spin echo sequence (TR/TE $=5000 \mathrm{~ms} /$ $10 \mathrm{~ms}, 16$ echoes, 10 coronal slices, slice thickness $0.5 \mathrm{~mm}$, FOV $2.5 \times 2.5 \mathrm{~cm}^{2}$, matrix $256 \times 256$, resolution $98 \times 98 \mu \mathrm{m}^{2}$, bandwidth $75 \mathrm{kHz}$ ).

Quantitative T2 maps were calculated from the multi-echo trains using the IDL software (Exelis Visual Information Solutions, Boulder, CO, USA), by pixelwise fitting signal intensities to a mono-exponential decay curve. Average T2 relaxation of the healthy cortex and striatum was determined. For determination of the lesion volume, all pixels on the ipsilateral hemisphere were counted with T2 values above the threshold of normal $\mathrm{T} 2+2$ standard deviations.

\section{Immunohistochemistry}

Animals were allowed to survive for 6 or 14 days after MCAO and were subsequently transcardially perfused under deep Isoflurane anesthesia with $20 \mathrm{ml}$ cold phosphate buffered saline (PBS), followed by $20 \mathrm{ml} 4 \%$ paraformaldehyde (PFA). Afterwards, brains were post-fixed in $4 \%$ PFA overnight and then cryo-protected by immersion in $30 \%$ sucrose solution for the next 2 days at $4{ }^{\circ} \mathrm{C}$. Then, brains were frozen in $-40{ }^{\circ} \mathrm{C}$ methylbutane (Sigma-Aldrich, Taufkirchen, Germany) and 
subsequently stored at $-80^{\circ} \mathrm{C}$. The brains were cut into $10 \mu \mathrm{m}$ coronal slices using a cryostat (Leica Microsystems, Wetzlar, Germany), directly mounted, and stored at $-20{ }^{\circ} \mathrm{C}$.

At the day of immunostaining the cryosections were kept at room temperature (RT) for $30 \mathrm{~min}$, and for stainings of ionized calcium-binding adapter molecule 1(Iba-1) acetone pretreatment at $-20{ }^{\circ} \mathrm{C}$ for 20 min was performed. To prevent non-specific binding of antibodies, the sections were preincubated in $5 \%$ normal serum and $0.25 \%$ Triton X-100, in potassium phosphate buffered saline (KPBS) for $60 \mathrm{~min}$ at RT. The treated sections were incubated overnight at $4{ }^{\circ} \mathrm{C}$ with subsequent primary antibodies: rabbit polyclonal anti Iba-1 (1:1000, 019-19,741; Wako Chem, Osaka, Japan), mouse anti-mannose receptor, MMR/CD206, (1:200, AF2535; R \& D Systems, Minneapolis, MN, USA) and rat anti CD16/32 (1:200, 101,301, Biolegend, San Diego, CA), followed by Cy5 and Cy 3 conjugated secondary antibodies (1:200, Jackson Immuno Research, West Grove, PA, USA) for $2 \mathrm{~h}$ at RT. For nuclear staining Hoechst 33,342 (1:1000; Invitrogen, Carlsbad, USA) was added together with secondary antibodies. Negatively stained control sections were included with equal preparation, excluding primary antibodies.

Three sections per mouse were imaged with a fluorescent microscope (BZ-9000 Keyence, Osaka, Japan) with 4×, 20× and $40 \times$ magnification objectives. 6 different regions of interest (ROIs) in each brain section were selected with the exposure time kept constant: the border and the core region of the ischemic hemisphere in the cortex and the striatum, further, two ROIs in the cortex and striatum of the intact hemisphere.

\section{Quantitative Immunohistochemical Analysis}

To observe the polarization of microglia/macrophages, immunofluorescence images were analyzed using NIH ImageJ analysis software (ImageJ) and TissueQuest 4.0 (TissueGnostics, Vienna, Austria), a specific image cytometry analysis software. Cells were identified on the tissue section based on the nuclei staining (nuclei size, staining intensity and discrimination by area was optimized manually) followed by the analysis of specific staining. To achieve optimal cell detection the background threshold was defined and due to the coverage of Iba-1 cell ramification with total nuclei intensity, the cutoffs were defined for each ROI to discriminate false signals. Scattergrams were created to visualize the corresponding positive cells in the source ROI through the real-time back gating component. Mean intensity and the relative number of coexpressed Iba-1 and CD206 or CD16/32 were obtained, and mean values were estimated from analyses of at least three brain sections per mouse. To observe M1 and M2 representative markers in double staining with Iba- 1 , the above-defined 6 different ROIs provided the following number of cells $/ \mathrm{mm}^{3}$ : i) total cell density according to the nuclei staining, ii) $\mathrm{Iba}-1^{+}$ microglial/macrophage cell density, iii) $\mathrm{CD} 206^{+} / \mathrm{Iba}-1^{+}$cell density, iv) $\mathrm{CD} 16 / 32^{+} / \mathrm{Iba}-1^{+}$cell density. Then, a mean was calculated for each region for each animal.

\section{Statistics}

Data were analyzed by SPSS version 22 (IBM SPSS statistics, Ehningen, Germany). The Normality test and homogeneity of variances were evaluated for all data. For behavioral scores (mNDS) the nonparametric analysis approach, Kruskal-Wallis $\mathrm{H}$, was performed. IHC data from early time point of liposomated miR-124 injection at day 2 were tested for significant changes between the 3 groups using one-way analysis of variance (ANOVA) with Bonferroni corrected posthoc comparisons. Using an independent one-tailed Student's $t$-test, we tested the data of late liposomated miR-124 injection to observe the significant changes between the stroke-only group and the group treated with miR-124 at day 10 .

The IHC numbers are represented by box-and-whisker plots (Figs. 3, 4 and 6) wherein each box shows the central $50 \%$ of the data points, the interquartile range (IQR), a horizontal line in each box indicates the median, and the vertical bars speak for the spread of $1.5 \times \mathrm{IQR}$. Dots display outliers, which were included in calculations of significance. The boxand-whisker plots were generated by using SPSS version 22 .

Bivariate correlation analysis between M1/M2 expression by Iba- $1^{+}$cells and mNDS were measured with Spearman's correlation coefficient. Regression was done with M1/M2 expression by Iba- $1^{+}$cells as the independent variable and mNDS as the dependent variable. A $p$-value $\leq 0.05$ was regarded statistically significant.

\section{Results}

\section{Characterization of Ischemic Lesion after Ischemia}

Two characteristic lesion types were generated 1) lesions restricted to the striatum and 2) lesions involving both striatum and cortex. We excluded the striatal-only ischemic mice from further experiments and used only a homogeneous group of cortico-striatal lesions. Quantitative MRI T2 maps of the included animals were acquired before stroke induction, and at day 2 and day 6 , respectively at day 2 and day 14 after MCAO, for experimental protocol 1 and 2. Thus the acute and chronic ischemic lesion location, the size and the development were detected (Fig. 1). One week before MCAO, T2 maps showed no signs of lesion and the healthy subjects presented equal $\mathrm{T} 2$ values of both hemisphere. At day 2 after MCAO, areas with markedly increased $\mathrm{T} 2$ values showed the development of the lesion extent over time. A gradual decrease in lesion volume was visible on the T2 maps at day 6 or day 14 (Fig. 1), showing that the vasogenic edema continuously resolved. A quantitative analysis of the lesion 
Fig. 1 Characterization of ischemic lesion after ischemia. Representative T2map of three experimental groups subjected to MCAO and perfused at day 6 or day 14 , respectively. T2 maps are shown as coronal brain section. T2 map of intact subjects, 1 week before MCAO, shows equal intensity of both hemispheres. $48 \mathrm{~h}$ after MCAO the cortico-striatal lesions are visible in the right hemisphere in

T2-weighted images. A gradual shrinkage of infarct volume was visible at days 6 and 14 compared to day 2 , showing the continuous reduction in vasogenic edema after stroke
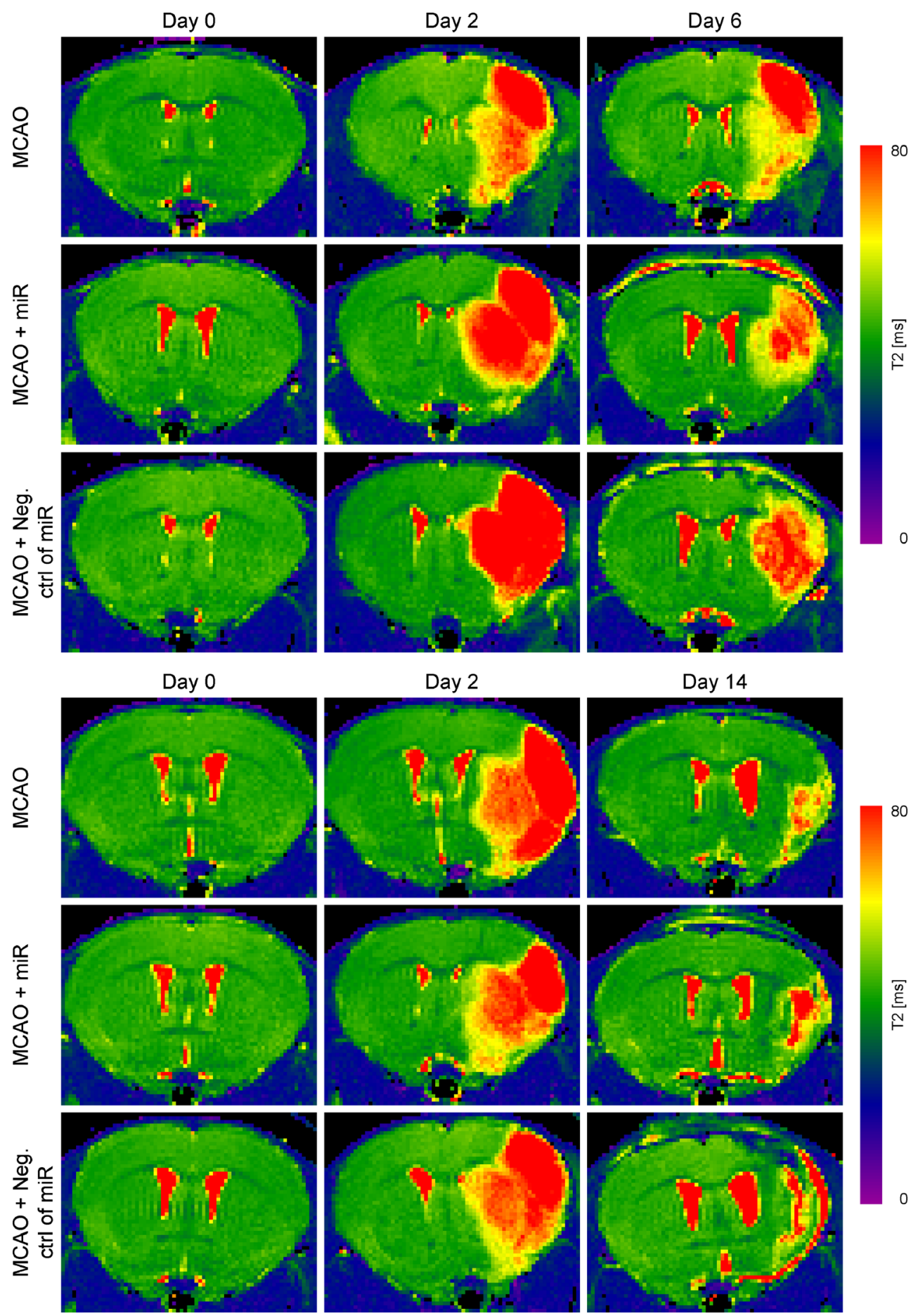

volume based on the elevated $\mathrm{T} 2$ relaxation time in the ischemic territory showed no significant difference between groups for all three experimental protocols (Suppl. Fig. 1).

\section{Morphology of Microglial/Macrophage after Stroke}

First we examined the morphology of ionized calciumbinding adapter molecule 1(Iba-1) positive immune cells in the stroke core, the border and the surrounding healthy tissue. Iba-1 detects CNS resident microglia and infiltrating monocyte-derived macrophages.

Iba-1 positive cells were visible in both intact and ischemic hemisphere, although with different morphology and distribution. They were densely gathered in the ischemic hemisphere around and inside the lesion (Fig. 2a). Microglia/macrophages were categorized into ramified, intermediate, amoeboid or 
Fig. 2 Different morphology and kinetics of microglial/ macrophage after stroke. The photomicrographs show the localization of Iba-1 positive cells intensely at the ipsilateral ischemic hemisphere at day 6 after stroke in $4 \times$ magnification (a), and the different morphology of microglial/macrophage cells classified into ramified, intermediate, amoeboid or round phenotype in $20 \times$ magnification (b). Representative graph shows the number of Iba-1 positive cells in the core and border zone of cortex and striatum at day 6 in comparison to day 14 after stroke (c). A significant increase in Iba-1 cell number was detected at day 6 after the onset of stroke. $n=5$ mice for each time point. Median and minimum/maximum values are shown; $* \mathrm{P}<0.05$ at independent one-tailed Student's t-test. Scale bar $50 \mu \mathrm{m}$

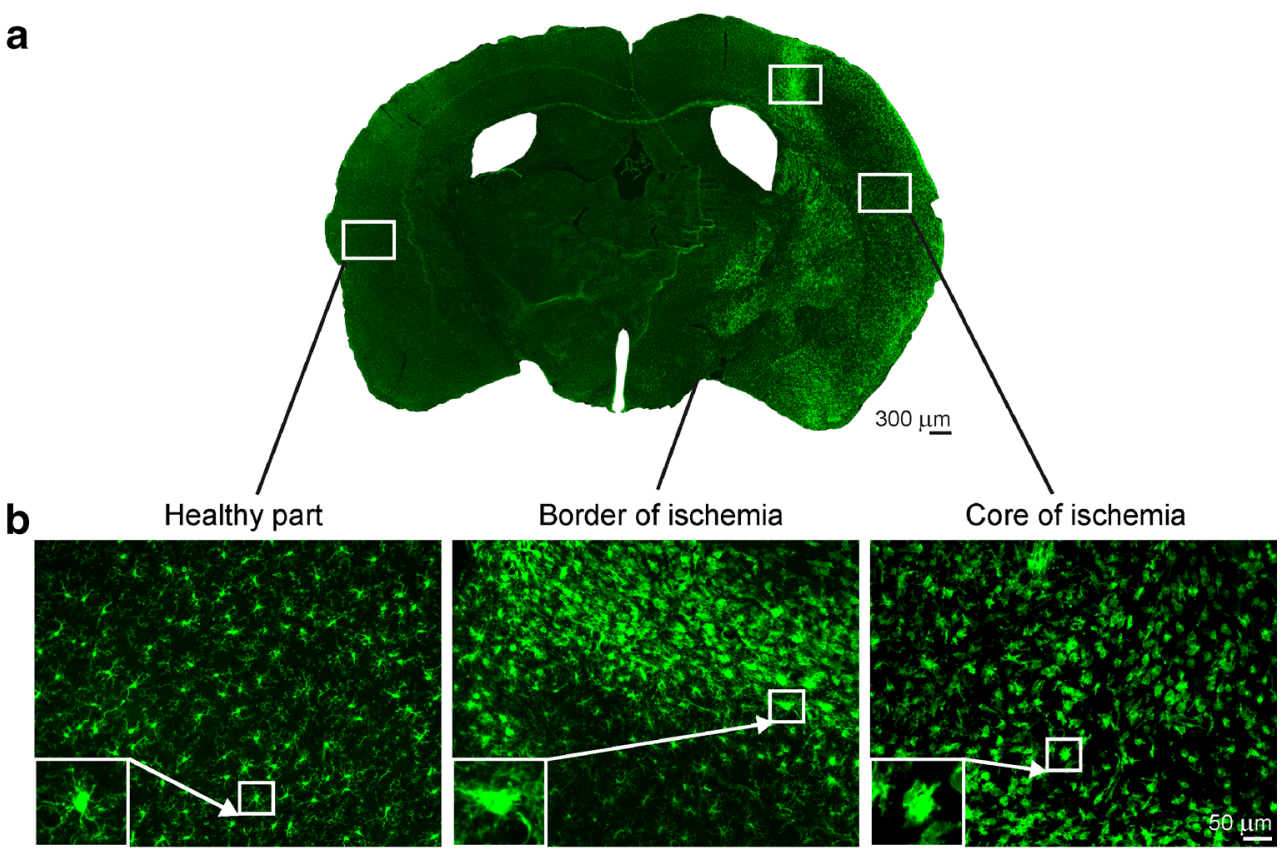

c

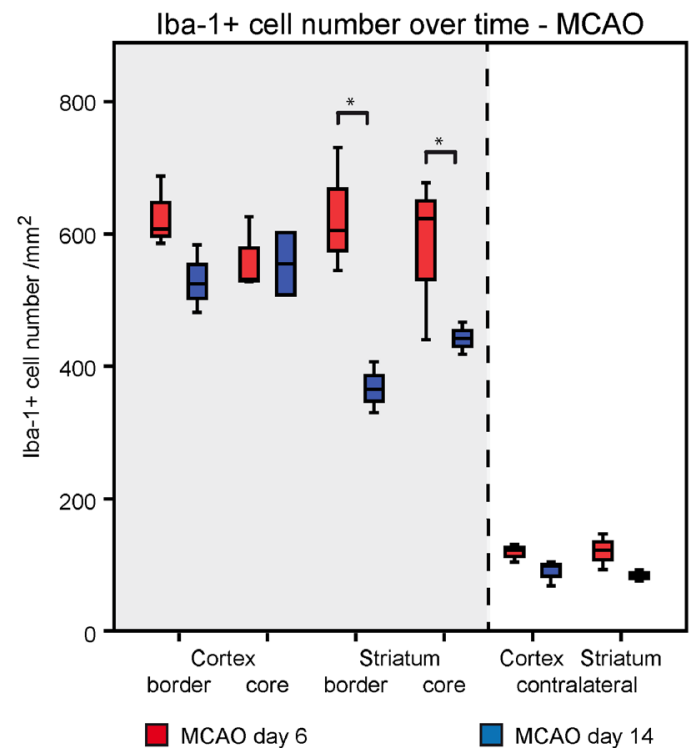

round phenotypes. The ramified type with long thin branching processes and a small cell body was mostly observed in the contralateral hemisphere and in the healthy parts of the ipsilateral ischemic hemisphere. In the peri-infarct area, the majority of Iba- $1^{+}$cells acquired the intermediate morphology with short or long swollen processes and amorphous larger cell body. In contrast, amoeboid and round shaped Iba- $1^{+}$cells were exclusively found in the ischemic core (Fig. 2b).

We noted a pronouncedly higher Iba- $1^{+}$cell density in the striatum at day 6 when compared with the results of day 14 post stroke (Fig. 2c), emphasizing the increased early stage proliferation of Iba- $1^{+}$cells after stroke.

\section{miR-124 Effects on Microglia/Macrophages after Stroke}

We further evaluated the influence of liposomated miR-124 (injection at day 2 after MCAO) on the expression of Iba- 1 at different time points after MCAO (Immunohistochemical analysis at day 6 Fig. 3a and day 14 Fig. 3b). Intracerebral injection of miR-124 at day 2 after stroke led to a significant increase in the number of Iba-1 cells. No significant difference in the number of Iba-1 positive cells was found between the MCAO-only and negative control group. At day 6 post $\mathrm{MCAO}$, all ipsilateral interquartile ranges of Iba- $1^{+}$ cell numbers were higher in the miR-124 treated group 


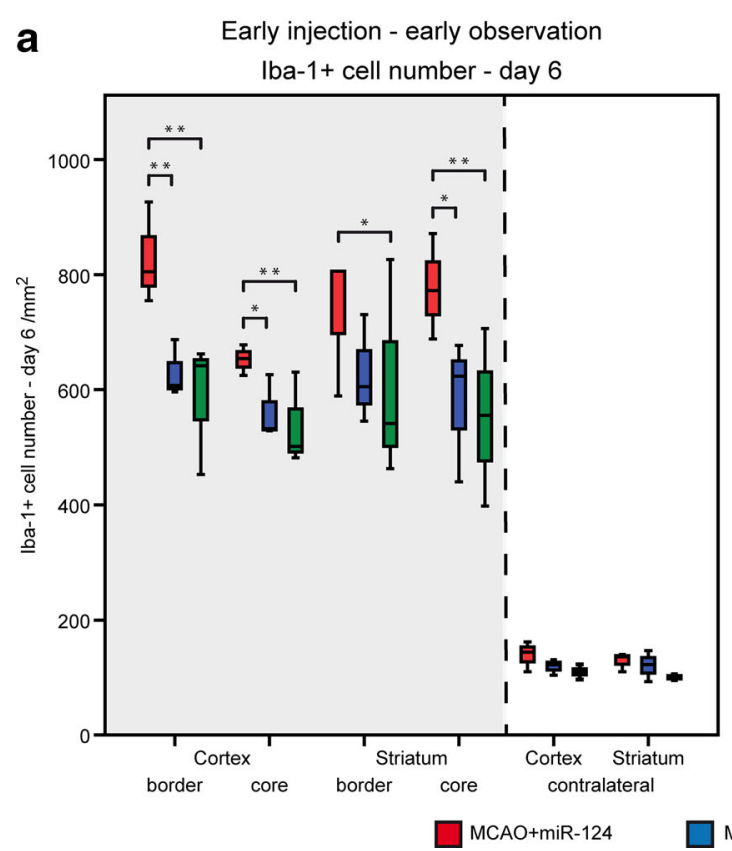

Fig. 3 miR-124 effects microglial/macrophage kinetic after stroke. Densities of Iba-1 positive cells in the border and core zone of cortex and striatum area in miR-124 treated group comparing to the only MCAO and negative control group at day 6 (a) and day 14 after stroke (b). A significant increase in Iba-1 cell number in miR-124 treated mice was

compared to both, the MCAO-only and the negative control group (Fig. 3a).

To observe the long term effects of the early injection of miR-124 on microglia/macrophage cells after stroke, we evaluated the Iba-1 cell number of the second experimental time point, where miR-124 or the random primer was injected 2 days after MCAO and animals were perfused at day 14 for immunohistochemical analysis. The density of Iba- $1^{+}$cells at day 14 markedly decreased in all 3 groups in all ipsilateral regions of interest, in comparison to the situation at day 6 post stroke (Fig. 3a, b). The Iba- $1^{+}$cell number in the cortical ROIs are at closely the same level in the three experimental groups (Fig. 3b).

\section{Modulation of Microglia/Macrophage Polarization by Early miR-124 Application}

Microglia/macrophages exhibit dynamic polarization over time, transforming from alternatively activated M2 phenotype to classical, activated M1 phenotype. We evaluated the characteristic polarization of microglia/macrophages after MCAO, using the representative M1-associated (CD16/32) or M2associated (CD206) markers for double immunofluorescent staining together with Iba-1 in the ischemic territory (Fig. 4 for CD206; Fig. 5 for CD16/32). In both, the MCAO-only and negative control group, the expression of M1 marker CD16/32 in Iba- $1^{+}$cells was high at day 6 after stroke and remained elevated until day 14 after ischemia (Fig. 5d, e). In contrast,

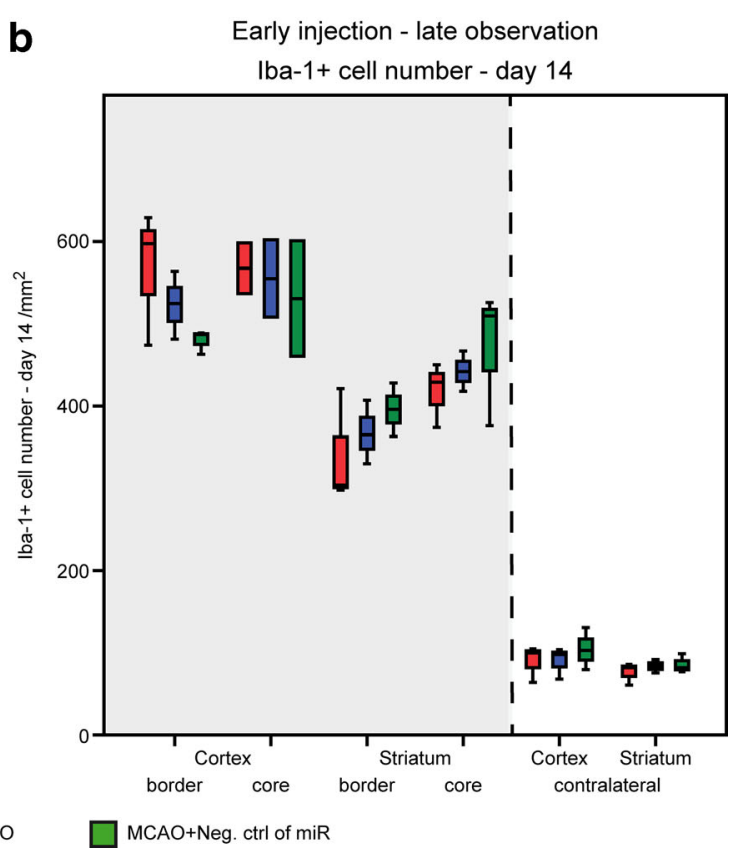

detected at day 6 after stroke. No significant difference in number of Iba-1 positive cells was found between the groups at day 14 after stroke. $n=5-$ 6 mice in each group and for each time point. Median and minimum/ maximum values are shown; $* \mathrm{P}<0.05, * * \mathrm{P}<0.01$ at one-way ANOVA (with Bonferroni's post hoc test)

the immunofluorescence for the M2 marker CD206 was slightly decreasing over time from day 6 until day 14 in both groups (Fig. 4d, e).

Intracerebral administration of $100 \mathrm{ng}$ miR-124 at day 2 after MCAO resulted in a strongly significant increase of Iba$1^{+}$cells expressing CD206 at day 6 post stroke. Interestingly, at this time point, the M2 marker expression tends to be higher in the border areas than in the core of the ischemic lesion in the miR-124 treated group, emphasizing the anti-inflammatory role of miR-124 in the penumbra (Fig. 4d). However, in comparison of the three experimental groups, no significant longterm effects of miR-124 on M2 marker expression were noted, although the miR treated group showed larger interquartile range in the cortex areas of the ischemic hemisphere indicating a trend towards a higher amount of CD206 expression by Iba- $1^{+}$ cells at day 14 after MCAO (Fig. 5e, Suppl. Fig. 2).

The immunofluorescence staining for M1 marker CD16/32 on Iba- $1^{+}$cells decreased significantly in the miR-124 treated group compared to both control groups at day 6 after MCAO. Almost all the relevant 50th percentile and the middle $50 \%$ of the ipsilateral ROIs of this group were in lower level, indicating a noticeably lesser pro-inflammatory environment in this miR-124 treated group (Fig. 5d). With miR-124 treatment the M1 response increased strongly from 6 days to 14 days after MCAO, indicating the decreasing effect of miR-124 twelve days after injection (Fig. 5e).

Consequently, the early intracranial injection of miR-124 had a significant effect on the ratio of anti-inflammatory to 

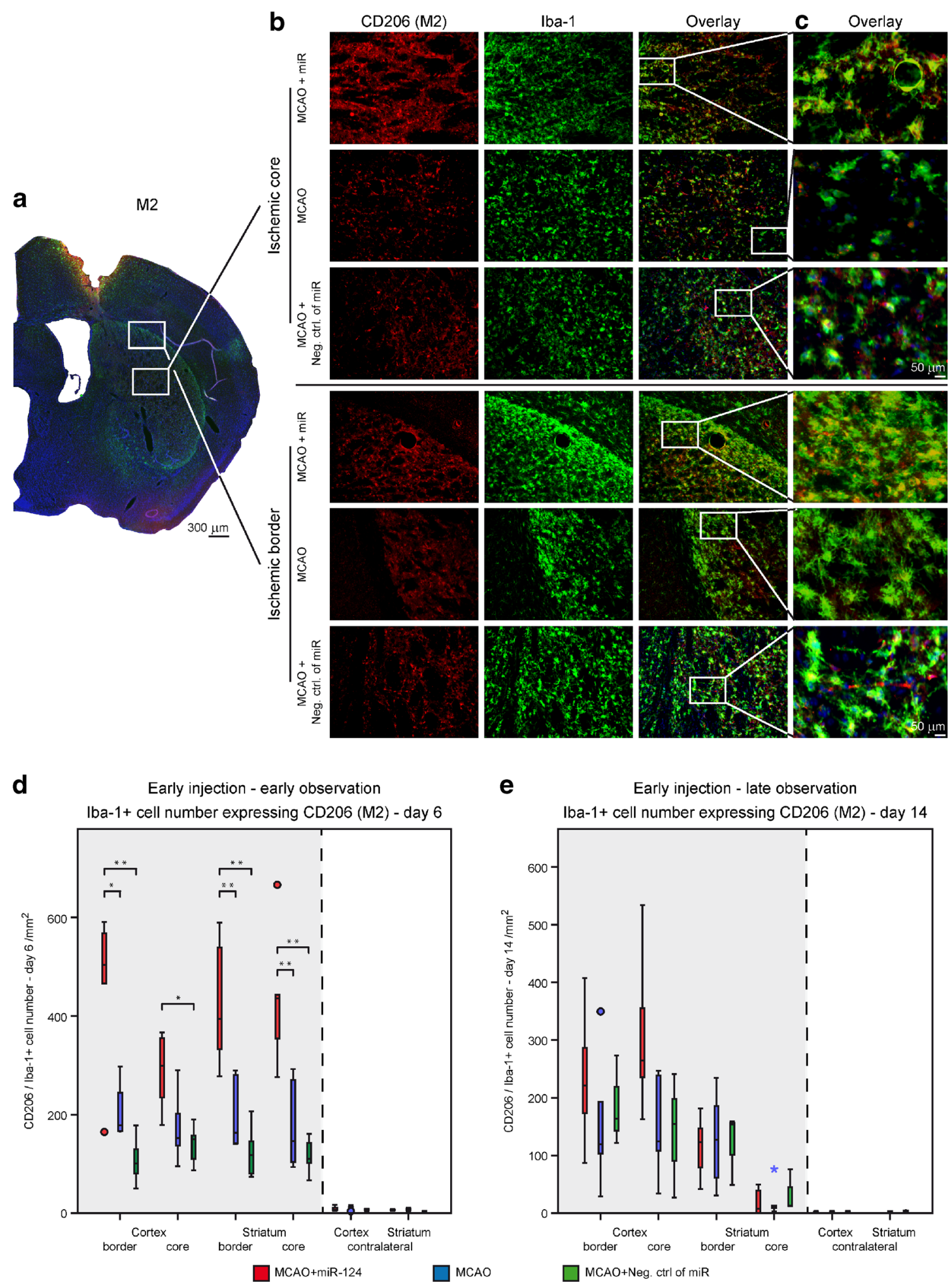

e

Early injection - late observation

Iba-1+ cell number expressing CD206 (M2) - day 14

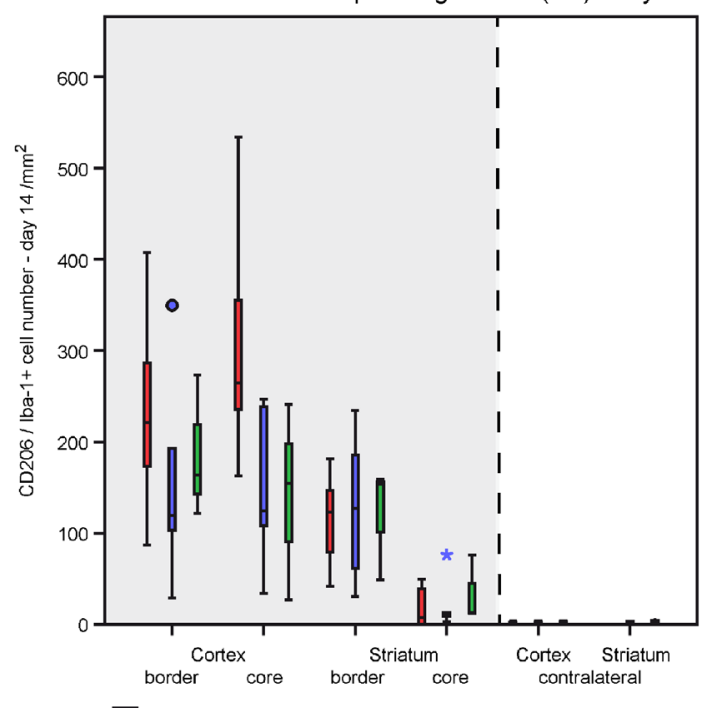

$\square \mathrm{MCAO}+$ Neg. ctrl of miR

Fig. 4 Effect of miR-124 on M2 phase of microglia/macrophages. Photomicrographs show examples of double-staining immunofluorescence of CD206 and Ibal on brain sections of ischemic core and border zones acquired from three experimental groups at 6 days after stroke. Illustrative $4 \times, 20 \times$ close-up magnification visualize the changes in CD206 expression by Iba-1 positive cells (a-c). Scale bar: $50 \mu \mathrm{m}$. Time course for the CD206 expression by Iba- $1+$ cells in the ipsilateral and contralateral ROIs of cortex and striatum in miR-124 treated group comparing to the only MCAO and

negative control group at day $6(\mathbf{d})$ and day $14(\mathbf{e})$. Quantification results confirm that intracranial injection of miR-124 at day 2 after MCAO results in a vigorous increase of Iba- $1+$ cells expressing CD206 monitored at day 6 post stroke (d). Representative graph shows no differences of CD206 expression by Iba-1+ cells at day 14 after stroke between groups (e). $\mathrm{n}=5-6$ mice in each group and for each time point. $* \mathrm{P}<0.05$, $* * \mathrm{P}<0.01$ at one-way ANOVA (with Bonferroni's post hoc test). Scale bar $50 \mu \mathrm{m}$ 
pro-inflammatory phenotype in the first week after MCAO (Fig. 7).

\section{Effects of Delayed miR-124 Injection on M1/M2 Phenotype of Microglia/Macrophages}

We investigated whether miR-124 application can also shift between the M1 and M2 phenotype at a later time after stroke when the pro-inflammatory M1 phenotype has already become dominant. To survey its effects at this later stage of stroke evolution, miR-124 was injected 10 days after MCAO, and the polarization of microglia/macrophages was analyzed 14 days after MCAO. No significant difference was observed in the M2 expressing Iba- $1^{+}$cell number between the miR-124 treated and the stroke-only group (Fig. 6e). The median of both groups did not differ in the cortical regions, however the 50th percentile of the ipsilateral striatal regions of miR-124 treated group were higher, pointing towards slightly increased M2 marker expression by $\mathrm{Iba}-\mathrm{1}^{+}$cells adjacent to the injection channel (Fig. 6e). Interestingly, a pronouncedly lower level of M1 marker expression by Iba- $1^{+}$cells was clearly visible in the miR-124 treated group, particularly in the border and core regions of the striatal lesion (Fig. 6f). The coherent interquartile range and the 50th percentile of all ipsilateral ROIs in the miR-124 treated group are at a remarkably lower level confirming the reduced M1 to M2 ratio. Analyzing the M1:M2 ratio directly demonstrates a significantly lower ratio under miR-124 treatment in all experiments. While the M1:M2 ratio of the miR-124 treated group is increasing with time after application, indicating a slowly weakened treatment effect, it remains still significantly lower even when miR-124 is applied only after ten days (Fig. 7).

Taken together, we have demonstrated that the delayed miR-124 administration after stroke still can modify the microglia and macrophage polarization while early application of miR-124 administration after stroke is needed to achieve maximal increase of protective M2 in parallel with proinflammatory M1 decrease.

\section{Neurological Deficit Scores}

We assessed whether intracerebral application of liposomated miR-124 improves functional recovery after stroke in mice, using the modified neurological deficit scores (mNDS). In all groups, $30 \mathrm{~min}$ MCAO increased the neurological deficit scores (up to $8 \mathrm{mNDS}$ ) compared to the days before stroke (0 mNDS) indicating impaired motor and sensory functions. Injection of miR-124 at two days after MCAO significantly reduced mNDS scores at 4 and 6 days $(P 0.05 ; 6$ and 4 mNDS, respectively) compared with the stroke-only and negative control group ( $8 \mathrm{mNDS}$ ). Slight differences were observed between the stroke-only and negative control group, however the differences were not significant (Fig. 8a, b). The mice treated with liposomated miR-124 at day 10 showed no treatment effect on mNDS scores (Fig. 8c). Our findings clearly indicate that the neurological deficits caused by $30 \mathrm{~min}$ MCAO in mice can be improved by early miR-124 injection.

\section{Correlation of M1/M2 and NDS}

Liposomated miR-124 treatment resulted in improved functional recovery, which was paralleled by the observation of decreased CD16/32 (M1) and increased CD206 (M2) expression of microglia/macrophages. We subsequently performed Spearman correlation analysis of M1 and M2 expression with the neurological deficit scores (Fig. 8d, e). Correlation analysis showed a positive linear relation between CD16/32 expression by Iba-1 positive cells and an increased neurological deficit score at day 6 after stroke with a linear equation NDS $=2.777+0.011(\mathrm{CD} 16 / 32)\left(\mathrm{r}^{2}=0.530\right.$, $p=0.017$ ), (Fig. 8d). A negative linear correlation was found between CD206 expression by Iba-1 positive cells and an increased neurological deficit score at day 6 after stroke with an equation NDS $=8.455-0.009(\mathrm{CD} 206)$ $\left(\mathrm{r}^{2}=0.425, p=0.041\right)$, (Fig. 8e). However, there was no correlation at day 14 post stroke.

\section{Discussion}

The main finding of the present study is that local miR-124 injection after ischemic stroke improves the neurological score at day 4 and 6 . The improved motor and sensory functions are directly correlated to the number of M2-like and inversely correlated to the number of M1-like microglia/macrophages. Taken together, our data present miR-124 as a promising molecule to modulate the microglia/macrophage activation state, and subsequently to improve the stroke recovery.

\section{Early Administration of miR-124, 2 days after Ischemic Stroke}

In an effort to control the phenotypic shift of microglia/ macrophages as a therapeutic strategy for brain disorders, we present a previously unreported role of the brain specific microRNA-124 in modulating the polarization of microglia and infiltrating macrophages in the sub-acute phase of ischemic stroke. We show here for the first time that miR-124 administration at $48 \mathrm{~h}$ after ischemia, before the proinflammatory peak of stroke (Hu et al. 2012), changes the M1/M2 balance toward a more anti-inflammatory phenotype and improves functional recovery at the first week after middle cerebral artery occlusion. The early miR-124 treatment at day 2 after ischemia gave rise to a strong increase of Iba- $1^{+}$ cells expressing the M2 marker CD206 and a parallel 


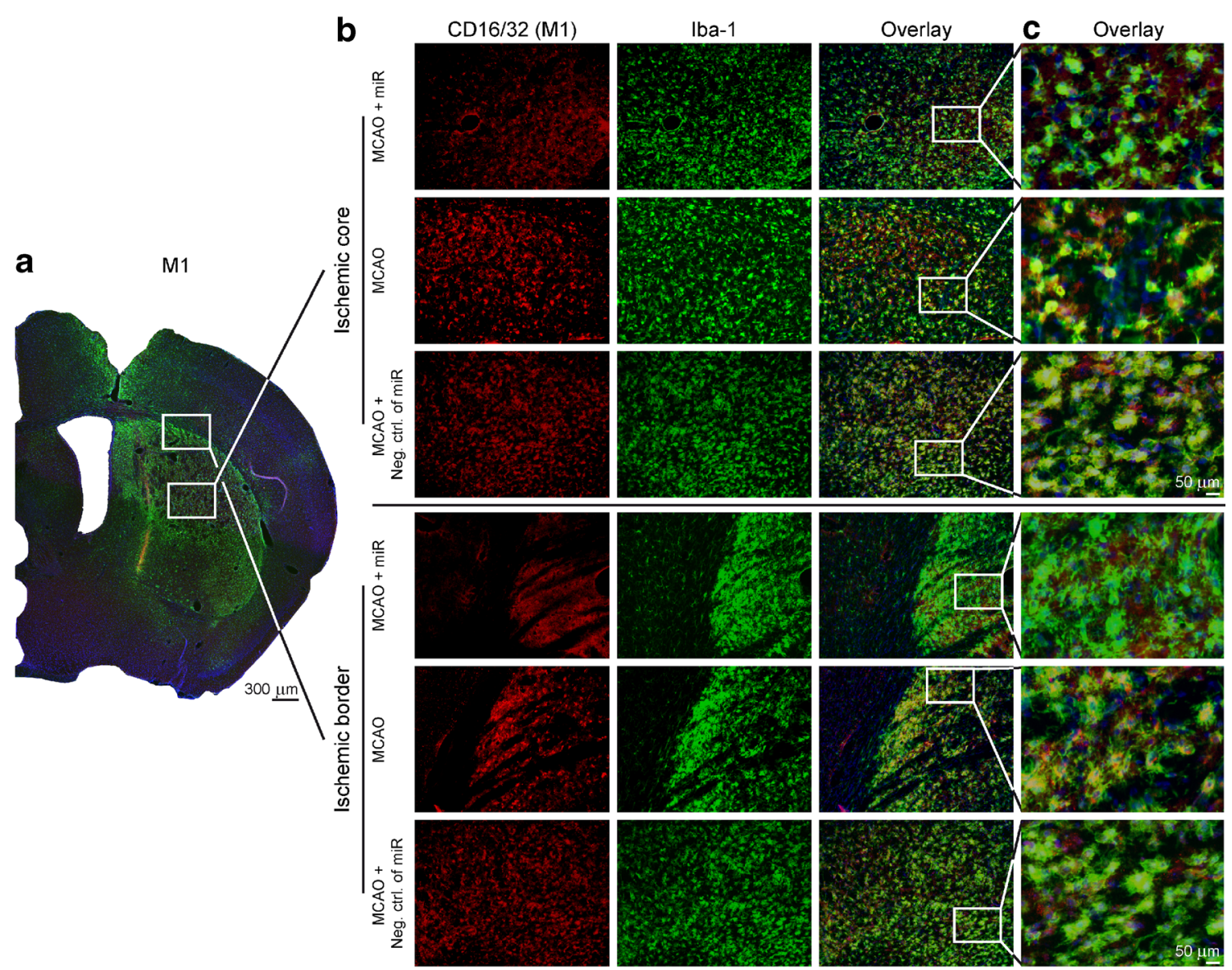

d

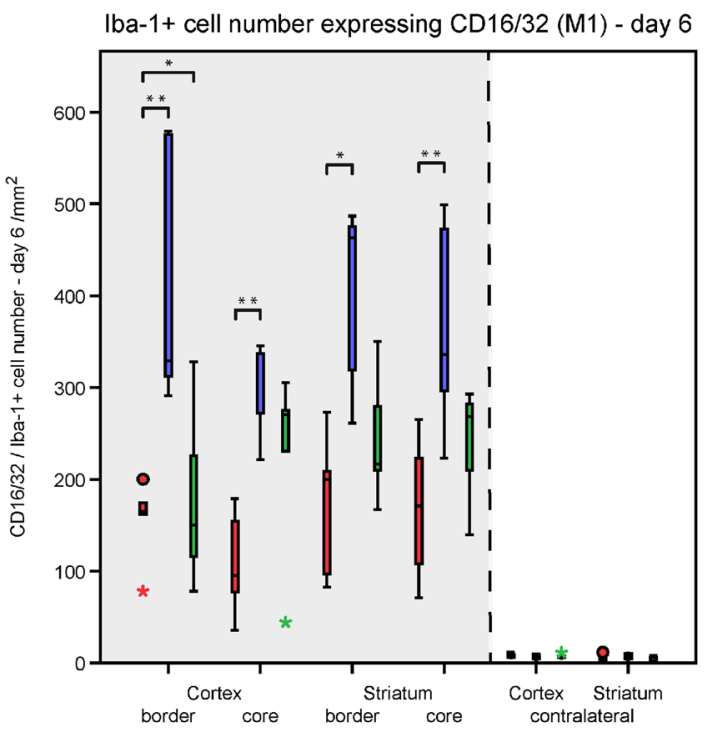

MCAO+miR-124 e

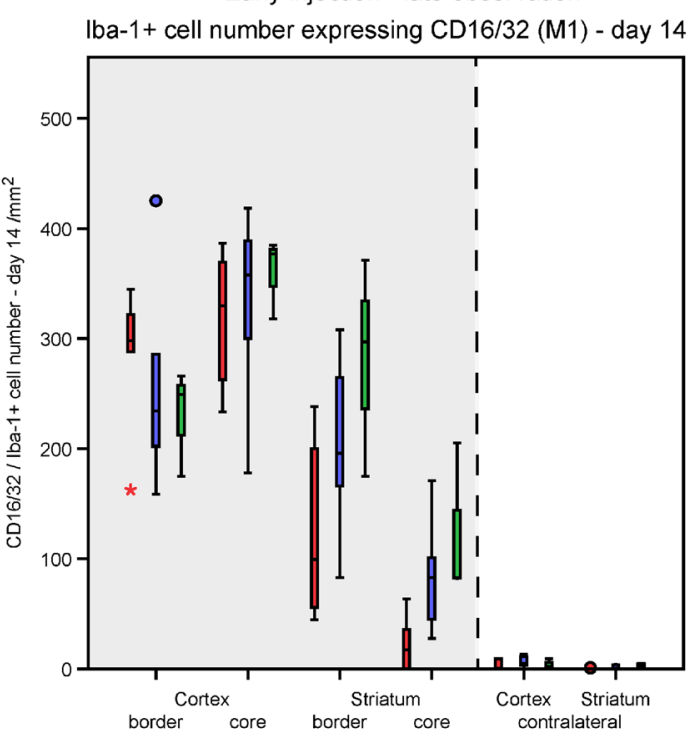

$\square \mathrm{MCAO}+\mathrm{Neg}$. Ctrl of miR pronounced decrease of the M1 marker CD16/32, highlighting the protective role of miR-124. In support of our experimental data, we observed a significant negative correlation between high M2 expression levels and neurological deficit scores in miR-124 administrated ischemic mice at day 6 , paralleled by a significant positive correlation of the M1 level with neurological deficit scores.

Microglia/macrophages react dynamically to ischemic injury over time: in the acute phase the vast majority of recruited microglia and macrophages in CNS diseases are M2-dominant, 
4 Fig. 5 Effect of miR-124 on M1 phase of microglia/macrophages. Photomicrographs show examples of double-staining immunofluorescence of CD16/32 and Iba- 1 on brain sections of ischemic core and border zones acquired from three experimental groups at 6 days after stroke. Illustrative $4 \times, 20 \times$ - close-up magnifications visualize the changes in CD16/32 expression by Iba-1 positive cells (a-c). Scale bar: $50 \mu \mathrm{m}$. Time course for the CD16/32 expression by Iba-1+ cells in the ipsilateral and contralateral ROIs of cortex and striatum in miR-124 treated group compared to the only MCAO and negative control group at day $6(\mathbf{d})$ and day 14 (e). Quantification results confirm that intracranial injection of miR124 at day 2 after MCAO results in a significant decrease of Iba-1+ cells expressing CD16/32 monitored at day 6 post stroke (d). Representative graph shows no differences at CD16/32 expression by Iba- $1+$ cells at day 14 after stroke between groups (e). $n=5-6$ mice in each group and for each time point. $* \mathrm{P}<0.05$, $* * \mathrm{P}<0.01$ at one-way ANOVA (with Bonferroni's post hoc test). Scale bar $50 \mu \mathrm{m}$

providing protective functions, but by about 1 week after injury the M1 phenotype becomes predominant (Kigerl et al. 2009; Perego et al. 2011; Wang et al. 2013). The mRNA expression of M2 phenotype markers (CD206, Arg-1, Ym1/2, IL-10, and TGF- $\beta$ ) peaks between day 3 to day 5 post injury, while the M1 phenotype relevant genes (iNOS, CD16, CD32) are continuously increasing over time from day 3 on. They remain elevated still at day 14 after stroke (Hu et al. 2012; Pan et al. 2015). This is in agreement with the expression peak of IRF-4 at day 3 , a key transcription factor of M2 polarization phenotype, whereas expression of IRF-8, a transcription factor of M1 polarization phenotype, increases within $72 \mathrm{~h}$ after ischemia (Guo et al. 2014; Xiang et al. 2014). The M2 phenotype promotes the CNS repair and regeneration, emphasizing that well timed counteraction of M2 on M1 should be a promising cell-based regenerative strategy in stroke.

We have shown that miR-124 enhances and prolongs the M2 phase while reducing the M1 process. Moreover, our results of improved functional outcome are in line with our earlier results, showing an upregulation of the M2-phenotype marker Arg-1 upon early administration of miR-124 after stroke (Hamzei Taj et al. 2016). In those studies, we could also find a negative correlation of Arg-1 expression with the neurological deficit score, paralleled by a positive correlation of Arg-1 cell expression with neuronal survival (Hamzei Taj et al. 2016). Further support for our finding of miR-124 induced improved functional outcome comes from Ponomarev (Ponomarev et al. 2011), who reported that bone marrow-derived macrophages, transfected with miR-124, downregulate the expression of the M1 markers TNF- $\alpha$ and inducible nitric oxide synthase (iNOS), and simultaneously upregulate the expression of the M2 markers TGF- $\beta 1$, Arg-1 and FIZZ1 (Found in inflammatory zone 1) by inhibition of the $\mathrm{C} / \mathrm{EBP}-\alpha$ transcription factor responsible for downregulation of PU.1. A similar M1/M2 ratio modulation of microglia and macrophages in the spinal cord was achieved via intrathecal injection of liposomated miR-124 in an IL-1 $\beta$ induced hyperalgesia model, leading to reduced inflammatory reactions and neuropathic pain (Willemen et al. 2012). When transfecting primary microglia isolated from neonatal rats with miR-124/chitosan complex particles, expression of TNF- $\alpha$ and inducible NO synthetase (iNOS), two major substances of M1 phenotype, was reduced. Microinjection of miR-124/chitosan particles was also shown to reduce the inflammation in a rat model of spinal cord injury (Louw et al. 2015). On the other hand, knockdown of miR-124 via a miR124 antisense oligonucleotide inhibitor was reported to activate the microglia and macrophages, presenting upregulated $\mathrm{MHC}$ class II and CD45 in addition to inhibiting the development of ramified morphology of the cells (Ponomarev et al. 2011).

It is worth noting that the induced modulation of immune response after stroke was mostly due to miR-124 injection in the present study and not because of intracranial injection procedure. Interestingly, however, the early injection of negative control of miR at day 2 after ischemia only gave rise to a smaller M1 marker expression by $\mathrm{Iba}-1^{+}$cells in comparison to the stroke only group. In support of our observation, Le Blon and colleagues observed fewer $\mathrm{MHCII}^{+}$expressions by $\mathrm{Iba}^{-} \mathrm{1}^{+}$cells, as M1 associated marker, in sham implanted group in a mouse model of mesenchymal stem cell implantation (Le Blon et al. 2014).

The M1/M2 modulating effect of the liposomated miR-124 was decreasing from day 6 to day 14 in our experiment. Although the number of CD206 $6^{+} / \mathrm{Iba}^{-}{ }^{+}$cells and CD16/32 ${ }^{+}$ Iba- $1^{+}$cells of the miR-124 treated group are no longer statistically significantly different from the corresponding cell number of the control groups, a clear difference is still noted resulting in the significantly lower M1:M2 ratio of the treated group (cf Fig. 7). We suggest that the time dependent decrease of the miR-124 immunomodulatory effect is explained by the short half-life of microRNAs of a few days only (Krol et al. 2010; Gantier et al. 2011). With a different route of miR-124 delivery the long term effect may be extended or even enhanced. Thus, Doeppner et al. presented sustained neuroprotection and ameliorated motor coordination and memory acquisition up to 56 days upon stroke onset by a viral vector-mediated miR-124 delivery (Doeppner et al. 2013). In correspondence to that, our findings of a decreasing immunomodulatory effect after a single injection of liposomated miR-124 together with the still persistent effect upon delayed injection indicates that future protocols with repetitive injections of liposomated miR-124 may provide a sustained low $\mathrm{M} 1: \mathrm{M} 2$ ratio.

\section{Delayed Administration of miR-124, 10 days after Ischemic Stroke}

We found that intracerebral injection of liposomated miR-124 at the chronic stage after stroke onset, administered at day 10 , still has substantial influence on the microglia/macrophages. However, while at early miR-124 application M2 was 

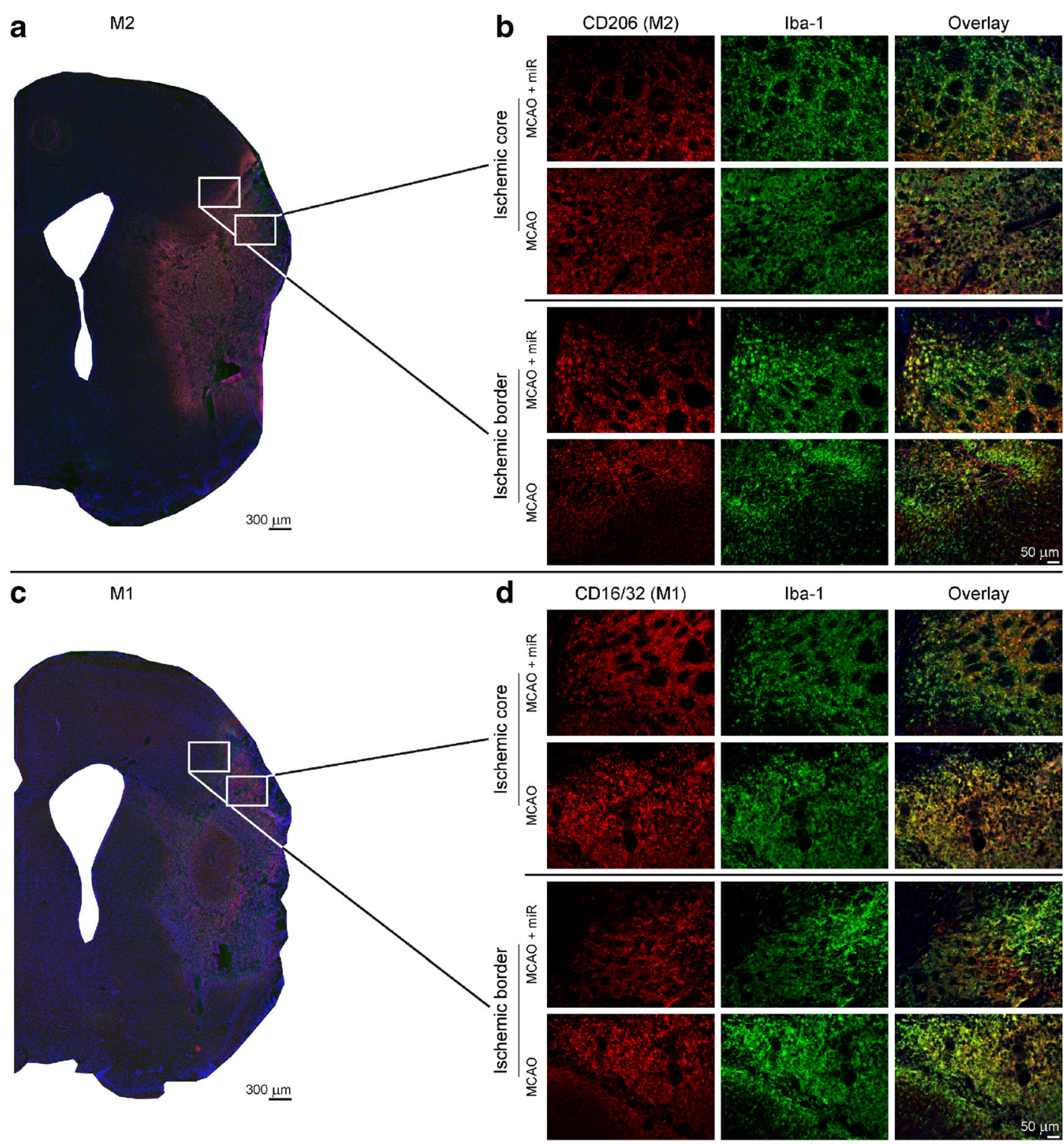

Overlay

e

Late injection - late observation

f Iba-1+ cell number expressing CD206 (M2) - day 14

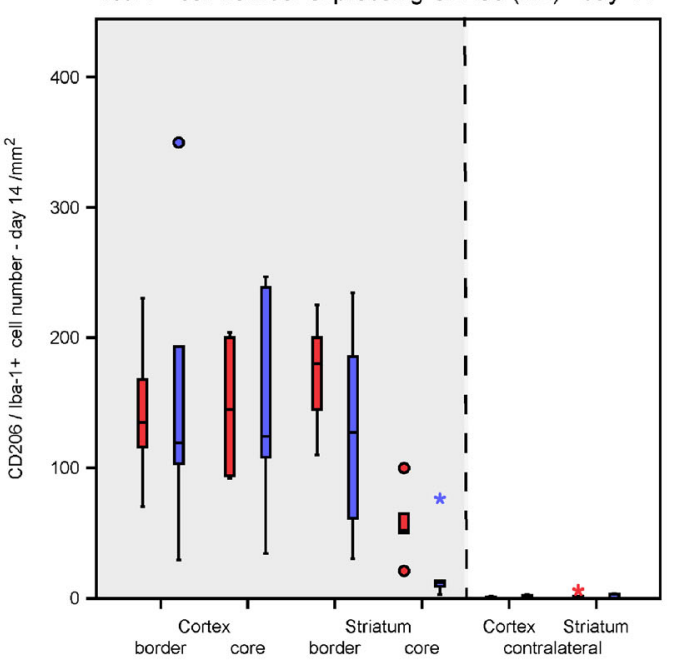

$\square$ MCAO+miR-124

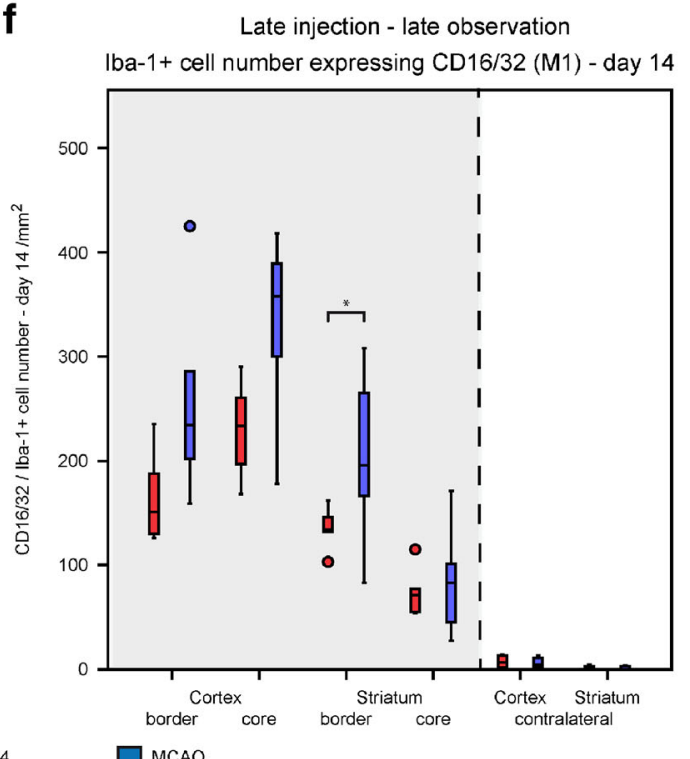


Fig. 6 Effects of delayed miR-124 injection on M1/M2 phenotype of microglia/macrophages. Photomicrographs show examples of doublestaining immunofluorescence of CD206 + Iba-1 (a-b) and CD16/32 + Iba-1 (c-d) on brain sections of ischemic core and border zones acquired from two experimental groups, where miR-124 was injected 10 days after MCAO and polarization of microglia/macrophages was analyzed 14 days after MCAO. Characteristic $4 \times$ and $20 \times$ magnifications display the changes in M2 and M1 markers expression by Iba-1 positive cells (a-d). Scale bar: $50 \mu \mathrm{m}$. Time course for the M2 (e) and M1 (f) markers expression by Iba- $1+$ cells in the ipsilateral and contralateral ROIs of cortex and striatum in miR-124 treated group comparing to the only MCAO group at day 14 . Quantification results show no significant difference in the M2 expressing Iba-1+ cells number between the miR124 treated and the only stroke groups (e). Representative graph confirms that intracranial injection of miR-124 at day 10 after MCAO results in pronounced decrease in M1 marker expression by Iba-1+ monitored at day 14 post stroke (f). $n=5$ mice in each group. $* \mathrm{P}<0.05$, independent one-tailed Student's t-test. Scale bar $50 \mu \mathrm{m}$

enhanced and M1 in parallel decreased, late phase application only decreased the pro-inflammatory M1 phenotype but left the M2 marker expression unaffected. This is in full agreement with our own earlier observation, where we showed that Arg-1, an M2 marker like CD206 used in the present study, was upregulated upon early miR-124 administration, but that this upregulation was lost when miR-124 was applied after ten days (Hamzei Taj et al. 2016). The miR-124 administration at this late stage when the pro-inflammatory phase has become dominant (Hu et al. 2012) could not improve the neurological deficit. Modulating the neuroinflammation in neurodegenerative disorders as a therapeutic strategy is only now emerging, and to our knowledge this is the first study to analyze the effect of polarization modulation of microglia/macrophage cells by liposomated miR-124 at different time points after stroke.

The M1 polarization process gradually increases from day 3 onward after stroke onset (Hu et al. 2012). We therefore addressed the question whether the delayed administration of miR-124 still has an effect on modifying the M1/M2 polarization. The strength of neuroinflammation modulation (i.e. $\mathrm{M} 1: \mathrm{M} 2$ ratio) in response to late miR-124 administration was lower compared to application at the early stage, which caused a pronounced increase in microglia/macrophages population with M2 phenotype and a noticeable decrease in the population with M1 phenotype. At this late time point, we observed a considerable decrease in M1 surface marker expression by Iba- $1^{+}$cells particularly in the border and core regions of the striatal lesion by miR-124 administration. Furthermore, the higher level of 50th percentile of the M2 surface marker expression of the ipsilateral striatal regions in the miR-124 treated group shows a small tendency of increased M2 surface marker expression by Iba- $1^{+}$cells at this time point. Consequently, even late miR-124 administration still has a clear, although somewhat weaker, modulatory effect on the M1-M2 balance by reducing the M1:M2 ratio, thus producing a clear, persistent shift towards the anti-inflammatory, neuroprotective microglia/macrophage phenotype. Intrathecal injection of liposomated miR-124 administration during the first $15 \mathrm{~h}$ of carrageenan-induced inflammatory hyperalgesia, when the pro-inflammatory process is prominent in this disease model, could effectively reduce the M1 surface marker and attenuated the resistant thermal hyperalgesia, although the efficacy of miR-124 on M1/M2 polarization at later time points of persistent hyperalgesia was not found (Willemen et al. 2012). Treatment with minocycline, a selective inhibitor of M1 microglia (Kobayashi et al. 2013), at day 4 after stroke, improved neurogenesis and functional recovery (Liu et al. 2007). These results suggest that targeting the correct phenotype of immune cells at the right time of neurodegenerative disorders is important for a therapeutic effect.

Our data demonstrate that miR-124 works in the early stage of stroke by modulating the microglia/macrophage activation
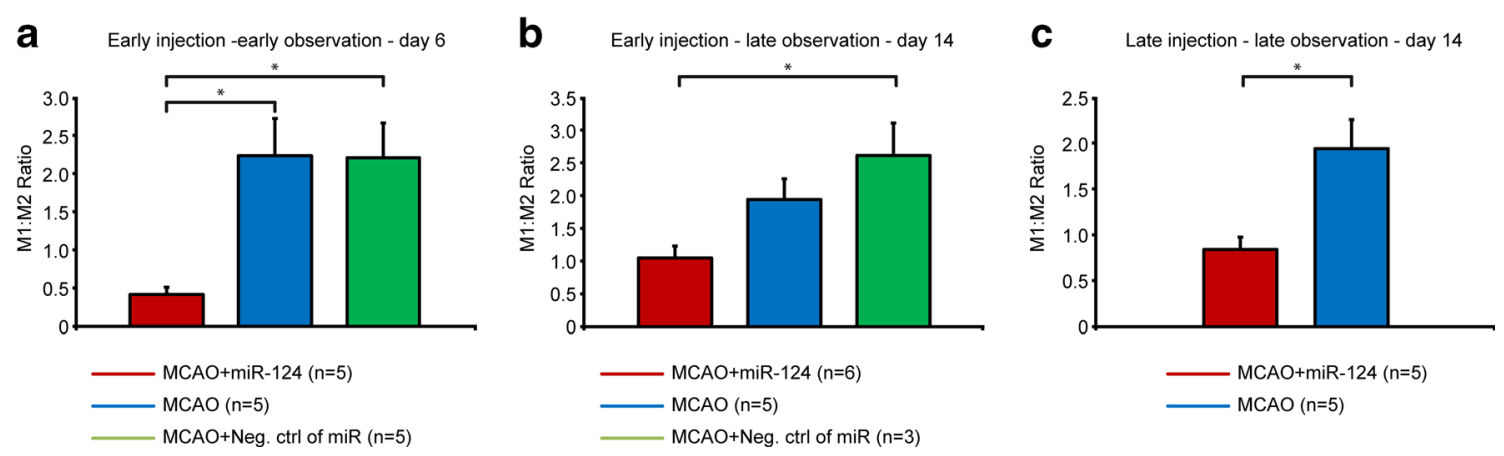

Fig. 7 M1:M2 ratio in the ischemic border zone of the striatum. The ratio of cell number with M1 (CD16/32) and M2 (CD206) phenotype was determined for the ischemic border zone of the striatum for all three animal groups (mir-124 treated; negative control; MCAO-only). a and b show the data for miR-124 injection at day 2 after stroke and analysis at day 6 (a) and at day 14 (b). c depicts the situation for delayed miR-124 injection at day 10 and analysis 4 days later, at day
14. While the M1:M2 ratio of the miR-124 treated group is slowly increasing with longer period after injection and with later injection time after stroke, it remains always substantially lower than the control groups. Results are expressed as mean \pm SEM. $\mathrm{P}<0.05$, as compared with control groups using Kruskal-Wallis $\mathrm{H}$ test for (a) and (b) and independent one-tailed Student's t-test for (c) 
a Early injection - early observation

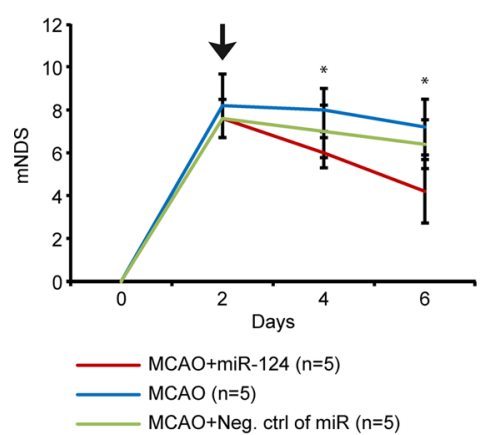

b Early injection - late observation

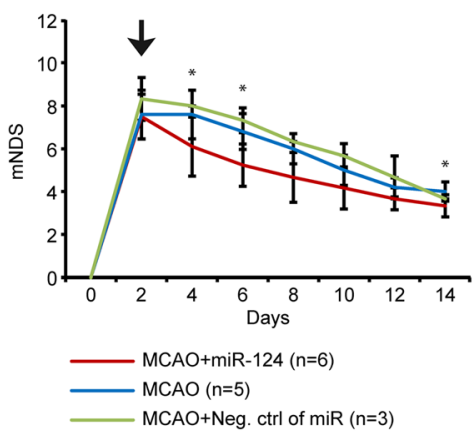

C Late injection - late observation

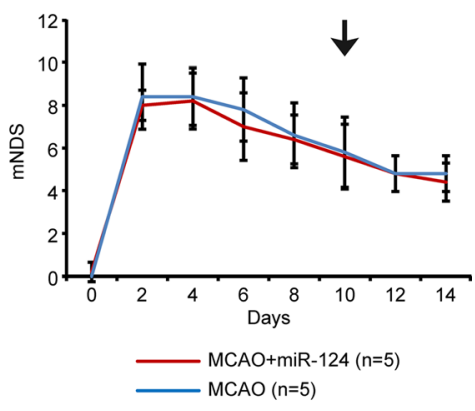

\section{Correlation - microglia/macrophage \& mNDS day 6}
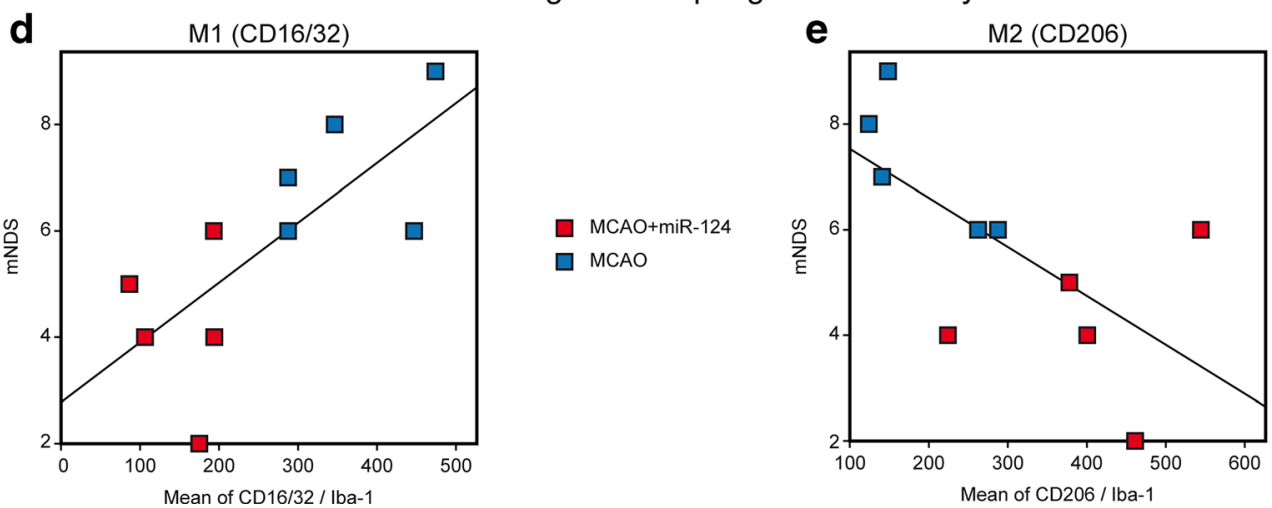

Fig. 8 MiR-124 improves functional recovery after stroke. Behavioral functional test was evaluated by the modified neurological deficit scores (mNDS) before and after MCAO. Representative graphs of the experimental groups subjected to $\mathrm{miR}$ transplantation at 2 days after MCAO and perfused at day 6 (a) and day 14, respectively (b) show that miR-124 treatment at 2 days after MCAO significantly improved the functional recovery at day 4 and day 6 after stroke. Representative graphs of the experimental group subjected to miR transplantation at day 10 after MCAO with perfusion at day 14 (c) show no significant

differences among the miR-124 treated group and only MCAO group. $\mathrm{n}=5-6$ mice in each group and for each time point. Values are mean $\pm \mathrm{SD}$. $* \mathrm{P}<0.05$, as compared with control groups using KruskalWallis $\mathrm{H}$ test for (a) and (b), and Wilcoxon-Mann-Whitney test for (c). Changes of M1 expression within the ischemic hemisphere linearly correlates with neurological deficit score changes in the ischemic mice (Spearman $\left.\mathrm{r}^{2}=0.530, p=0.017\right)(\mathbf{d})$. Changes of M2 expression within the ischemic hemisphere inversely correlates to neurological deficit score changes in the ischemic mice (Spearman $\mathrm{r} 2=0.425, p=0.041)(\mathbf{e})$

towards M2 phenotype, leading to improved functional recovery. Delayed miR-124 administration after stroke still modified the microglia and macrophages M1/M2 polarization ratio, however with reduced intensity. This indicates that a maximal effect is achieved for miR-124 administration before the dominance of the pro-inflammatory process of stroke, i.e. within the first few days when the anti-inflammatory phase of the M2 phenotype is dominant. Moreover, therapeutic effect of the long term observed polarization shift by miR-124 was limited to early application. It clearly emphasizes the necessity of careful adjustment of the polarization balance of microglia/macrophages in therapeutic approaches targeting neuroinflammation. Although much work is still needed before clinical translation, our findings provide important steps for the understanding of microRNA based therapy and the underlying mechanisms to develop microRNA injection into a new approach for functional improvement after ischemic stroke.

\section{Conclusions}

In conclusion, our study demonstrates that a single injection of miR-124 into the mouse brain in the early phase after stroke is sufficient to shift the M1/M2 balance of immune cells towards the anti-inflammatory phenotype which correlates with behavioral improvement. Further studies are needed to clarify the mechanism and identify the targeted immune cells. Nevertheless, our findings highlight the important role of immune cells after stroke and the therapeutic relevance of their polarization balance. As there is no treatment for sub-acute stroke available, miR-124 evolves as a promising novel therapeutic approach.

CCA, common carotid artery; C/EBP- $\alpha$, CCAAT enhancer binding protein $\alpha$; CNS, central nervous system; ECA, external carotid artery; FIZZ, found in inflammatory zone; Iba-1, ionized calcium-binding adapter molecule 1; ICA, internal carotid artery; IFN, interferon; IL, interleukin; iNOS, inducible 
NO synthetase; IQR, interquartile range; KPBS, potassium phosphate buffered saline; MCAO, middle cerebral artery occlusion; MHC, major histocompatibility complex; MMR, mouse anti-mannose receptor; mNDS, modified neurological deficit score; mRNA, messenger ribonucleic acid; MRI, magnetic resonance imaging; MSME, multi slice multi echo; PBS, phosphate buffered saline; PFA, paraformaldehyde; RF, radio frequency; RNA, ribonucleic acid; siRNA, small interfering ribonucleic acid; TGF- $\beta$, tumor growth factor $\beta$; TNF- $\alpha$, tumor necrosis factor $\alpha$.

Acknowledgments Open access funding provided by Max Planck Society. We thank Ulla Uhlenküken for professional arrangement of the graphics and Melanie Nelles for help with histology. This work was financially supported by grants from the EU-FP7 programs TargetBraIn (HEALTH-F2-2012-279017) and BrainPath (PIAPP-GA2013-612360)

\section{Compliance with Ethical Standards}

Funding This study was financially supported by grants from the EU 7th Framework Programme (grant numbers HEALTH-F2-2012-279017 and PIAPP-GA-2013-612360).

Conflict of Interest Mathias Hoehn received research grants from the European Union under the 7th Framework Programme.

Ethical Approval All animal experiments were conducted according to the guidelines laid out in the German Animal Welfare Act, in accordance with the European Council Directive 2010/63/EU, and were approved by the regional authorities (Landesamt für Natur, Umwelt und Verbraucherschutz North Rhine-Westphalia; reference number 84-0204-2011.A123).

Open Access This article is distributed under the terms of the Creative Commons Attribution 4.0 International License (http:// creativecommons.org/licenses/by/4.0/), which permits unrestricted use, distribution, and reproduction in any medium, provided you give appropriate credit to the original author(s) and the source, provide a link to the Creative Commons license, and indicate if changes were made.

\section{References}

Adamczak JM, Schneider G, Nelles M, Que I, Suidgeest E, van der Weerd L, Lowik C, Hoehn M (2014) In vivo bioluminescence imaging of vascular remodeling after stroke. Front Cell Neurosci 8:274

Aswendt M, Gianolio E, Pariani G, Napolitano R, Fedeli F, Himmelreich U, Aime S, Hoehn M (2012) In vivo imaging of inhibitory, GABAergic neurons by MRI. NeuroImage 62:1685-1693

Bartel DP (2009) MicroRNAs: Target Recognition and Regulatory Functions. Cell 136:215-233

Bhatnagar N, Ahmad F, Hong HS, Eberhard J, Lu IN, Ballmaier M, Schmidt RE, Jacobs R, Meyer-Olson D (2014) FcgammaRIII (CD16)-mediated ADCC by NK cells is regulated by monocytes and FcgammaRII (CD32). Eur J Immunol 44:3368-3379

Bi YJ, Liu GW, Yang RF (2009) MicroRNAs: Novel Regulators During the Immune Response. J Cell Physiol 218:467-472

Chen J, Sanberg PR, Li Y, Wang L, Lu M, Willing AE, Sanchez-Ramos J, Chopp M (2001) Intravenous administration of human umbilical cord blood reduces behavioral deficits after stroke in rats. Stroke 32:2682-2688

Dirnagl U, Iadecola C, Moskowitz M (1999) Pathobiology of ischemic stroke: an integrated view. Trends Neurosci 22:391-397

Dirnagl U, Klehmet J, Braun JS, Harms H, Meisel C, Ziemssen T, Prass K, Meisel A (2007) Stroke-induced immunosuppression. Experimental evidence and clincial relevance. Stroke 38:770-773

Doeppner TR, Doehring M, Bretschneider E, Zechariah A, Kaltwasser B, Muller B, Koch JC, Bahr M, Hermann DM, Michel U (2013) MicroRNA-124 protects against focal cerebral ischemia via mechanisms involving Usp14-dependent REST degradation. Acta Neuropathol 126:251-265

Donnan GA, Fisher M, Macleod M, Davis SM (2008) Stroke. Lancet $371: 1612-1623$

Ekdahl CT, Claasen JH, Bonde S, Kokaia Z, Lindvall O (2003) Inflammation is detrimental for neurogenesis in adult brain. Proc Natl Acad Sci U S A 100:13632-13637

Gantier MP, McCoy CE, Rusinova I, Saulep D, Wang D, Xu D, Irving AT, Behlke MA, Hertzog PJ, Mackay F, Williams BR (2011) Analysis of microRNA turnover in mammalian cells following Dicer1 ablation. Nucleic Acids Res 39:5692-5703

Guo S, Li ZZ, Jiang DS, Lu YY, Liu Y, Gao L, Zhang SM, Lei H, Zhu LH, Zhang XD, Liu DP, Li H (2014) IRF4 is a novel mediator for neuronal survival in ischaemic stroke. Cell Death Differ 21:888-903

Hamzei Taj S, Kho W, Riou A, Wiedermann D, Hoehn M (2016) MiRNA-124 induces neuroprotection and functional improvement after focal cerebral ischemia. Biomaterials 91:151-165

Hanisch UK, Kettenmann H (2007) Microglia: active sensor and versatile effector cells in the normal and pathologic brain. Nat Neurosci 10: $1387-1394$

Hirai T, Uchida K, Nakajima H, Guerrero AR, Takeura N, Watanabe S, Sugita D, Yoshida A, Johnson WE, Baba H (2013) The prevalence and phenotype of activated microglia/macrophages within the spinal cord of the hyperostotic mouse (twy/twy) changes in response to chronic progressive spinal cord compression: implications for human cervical compressive myelopathy. PLoS One 8:e64528

Hu X, Li P, Guo Y, Wang H, Leak RK, Chen S, Gao Y, Chen J (2012) Microglia/macrophage polarization dynamics reveal novel mechanism of injury expansion after focal cerebral ischemia. Stroke 43: 3063-3070

Hu X, Leak RK, Shi Y, Suenaga J, Gao Y, Zheng P, Chen J (2015) Microglial and macrophage polarization-new prospects for brain repair. Nat Rev Neurol 11:56-64

Jia J, Peng J, Li Z, Wu Y, Wu Q, Tu W, Wu M (2016) Cannabinoid CB2 Receptor Mediates Nicotine-Induced Anti-Inflammation in N9 Microglial Cells Exposed to beta Amyloid via Protein Kinase C. Mediat Inflamm 2016:4854378

Jin R, Yang G, Li G (2010) Inflammatory mechanisms in ischemic stroke: role of inflammatory cells. J Leukoc Biol 87:779-789

Kigerl KA, Gensel JC, Ankeny DP, Alexander JK, Donnelly DJ, Popovich PG (2009) Identification of two distinct macrophage subsets with divergent effects causing either neurotoxicity or regeneration in the injured mouse spinal cord. J Neurosci 29:13435-13444

Kobayashi K, Imagama S, Ohgomori T, Hirano K, Uchimura K, Sakamoto K, Hirakawa A, Takeuchi H, Suzumura A, Ishiguro N, Kadomatsu K (2013) Minocycline selectively inhibits M1 polarization of microglia. Cell Death Dis 4:e525

Krol J, Busskamp V, Markiewicz I, Stadler MB, Ribi S, Richter J, Duebel J, Bicker S, Fehling HJ, Schubeler D, Oertner TG, Schratt G, Bibel M, Roska B, Filipowicz W (2010) Characterizing light-regulated retinal microRNAs reveals rapid turnover as a common property of neuronal microRNAs. Cell 141:618-631

Kwon MJ, Kim J, Shin H, Jeong SR, Kang YM, Choi JY, Hwang DH, Kim BG (2013) Contribution of macrophages to enhanced regenerative capacity of dorsal root ganglia sensory neurons by conditioning injury. J Neurosci 33:15095-15108 
Le Blon D, Hoornaert C, Daans J, Santermans E, Hens N, Goossens H, Berneman Z, Ponsaerts P (2014) Distinct spatial distribution of microglia and macrophages following mesenchymal stem cell implantation in mouse brain. Immunol Cell Biol 92:650-658

Liu Z, Fan Y, Won SJ, Neumann M, Hu D, Zhou L, Weinstein PR, Liu J (2007) Chronic treatment with minocycline preserves adult new neurons and reduces functional impairment after focal cerebral ischemia. Stroke 38:146-152

Liu C, Li Y, Yu J, Feng L, Hou S, Liu Y, Guo M, Xie Y, Meng J, Zhang H, Xiao B, Ma C (2013) Targeting the Shift from M1 to M2 macrophages in experimental autoimmune encephalomyelitis mice treated with Fasudil. PLoS One 8:e54841

Louw AM, Kolar MK, Novikova LN, Kingham PJ, Wiberg M, Kjems J, Novikov LN (2016) Chitosan polyplex mediated delivery of miRNA-124 reduces activation of microglial cells in vitro and in rat models of spinal cord injury. Nanomedicine 12:643-653

Makeyev EV, Zhang J, Carrasco MA, Maniatis T (2007) The MicroRNA miR-124 promotes neuronal differentiation by triggering brainspecific alternative pre-mRNA splicing. Mol Cell 27:435-448

Miron VE, Boyd A, Zhao JW, Yuen TJ, Ruckh JM, Shadrach JL, van Wijngaarden P, Wagers AJ, Williams A, Franklin RJ, ffrenchConstant C (2013) M2 microglia and macrophages drive oligodendrocyte differentiation during CNS remyelination. Nat Neurosci 16: $1211-1218$

Pan J, Jin JL, Ge HM, Yin KL, Chen X, Han LJ, Chen Y, Qian L, Li XX, $\mathrm{Xu} Y$ (2015) Malibatol A regulates microglia M1/M2 polarization in experimental stroke in a PPARgamma-dependent manner. J Neuroinflammation 12:51
Patel AR, Ritzel R, McCullough LD, Liu F (2013) Microglia and ischemic stroke: a double-edged sword. International journal of Pysiology pathophysiology. Pharmacology 5:73-90

Perego C, Fumagalli S, De Simoni MG (2011) Temporal pattern of expression and colocalization of microglia/macrophage phenotype markers following brain ischemic injury in mice. J Neuroinflammation $8: 174$

Ponomarev ED, Veremeyko T, Barteneva N, Krichevsky AM, Weiner HL (2011) MicroRNA-124 promotes microglia quiescence and suppresses EAE by deactivating macrophages via the C/EBP-alphaPU.1 pathway. Nat Med 17:64-70

Thored P, Heldmann U, Gomes-Leal W, Gisler R, Darsalia V, Taneera J, Nygren JM, Jacobsen SE, Ekdahl CT, Kokaia Z, Lindvall O (2009) Long-term accumulation of microglia with proneurogenic phenotype concomitant with persistent neurogenesis in adult subventricular zone after stroke. Glia 57:835-849

Visvanathan J, Lee S, Lee B, Lee JW, Lee SK (2007) The microRNA miR-124 antagonizes the anti-neural REST/SCP1 pathway during embryonic CNS development. Genes Dev 21:744-749

Wang G, Zhang J, Hu X, Zhang L, Mao L, Jiang X, Liou AK, Leak RK, Gao Y, Chen J (2013) Microglia/macrophage polarization dynamics in white matter after traumatic brain injury. J Cereb Blood Flow Metab 33:1864-1874

Willemen HL, Huo XJ, Mao-Ying QL, Zijlstra J, Heijnen CJ, Kavelaars A (2012) MicroRNA-124 as a novel treatment for persistent hyperalgesia. J Neuroinflammation 9:143

Xiang M, Wang L, Guo S, Lu YY, Lei H, Jiang DS, Zhang Y, Liu Y, Zhou Y, Zhang XD, Li H (2014) Interferon regulatory factor 8 protects against cerebral ischaemic-reperfusion injury. J Neurochem 129: 988-1001 